\title{
CONTACT ANALYSES FOR ANISOTROPIC HALF SPACE WITH AN ANISOTROPIC COATING
}

\author{
C. Bagault \\ D. Nélias \\ M.-C. Baietto \\ Université de Lyon, CNRS, INSA-Lyon, LaMCoS UMR5259, F-69621, Villeurbanne, France \\ T. Ovaert \\ University of Notre Dame, Indiana, USA
}

\begin{abstract}
For most composite and mono-crystal materials their compositions or the elaboration and manufacturing processes imply that it exists one or two main directions or even a general anisotropy. Moreover, coatings are often used to prevent or control wear. Coatings do not have, generally, the same properties as the substrate and may have various thicknesses. The influence of the anisotropy orientations (in the coating and in the substrate) have to be taken into account to better predict the distribution of the contact pressure and the subsurface stress-field in order to optimize the service life of industrial components. A contact model using semi analytical methods, relying on elementary analytical solutions, has been developed. It is based on numerical techniques adapted to contact mechanics. Recent developments aim to quantify displacements and stresses of a layered anisotropic elastic half space which is in contact with a rigid sphere. The influence of material properties and layer thickness on the contact problem solution will be more specifically analyzed.
\end{abstract}

\section{INTRODUCTION}

Starting from three dimensional Green's functions in anisotropic trimaterials [1], these functions are derived for layered anisotropic half space, as a sum of infinite space Green's functions and a complementary part, similarly to the Mindlin's superposition method. The Green's functions for anisotropic infinite space have an explicit expression, given by Ting and Lee [2], whereas the complementary part needs to be integrated numerically. The terms of the complementary part convey to different order of image and can be reduced to different cases, as the bimaterial Green's functions with the first order.

A similar work has been done for the contact between a rigid sphere and an anisotropic half space [3], using the three dimensional Green's functions in anisotropic bimaterials [4]. It is found that the stiffness along the normal to the contact has a strong influence on the contact solution in terms of pressure distribution and contact size. Conversely a change of the Young's modulus along a direction parallel to the surface does not significantly affect the contact pressure distribution but the contact area is no more circular. The performance of the method is highlighted by analysing the effect of the orientation of the material main directions compared to the surface normal. $\mathrm{He}$ and Ovaert [5] also focused on anisotropic materials by studying the rough surface contact.

Several methods can be used for contact simulation of anisotropic materials. Semi analytical methods have proven their efficiency in contact mechanics and are developed here to account for anisotropy of materials. The main advantage is here the computing time compared to the Finite Element (FE) method that is however widely used for many contact problems. The contact model between an anisotropic material with an anisotropic coating and a rigid sphere will be presented.

\section{SOLUTION OF THE CONTACT PROBLEM}

The semi analytical method consists in the summation of elementary solutions known analytically. One of the difficulties is the derivation or the identification of these elementary analytical solutions, such as the well known Boussinesq and Cerruti solutions in isotropic elasticity [6]. The semi analytical contact solver is based on the pioneering work of Jacq et al. [7] for elastic-plastic contacts.

The frame is simplified here by supposing the contact between one anisotropic elastic half space and a rigid body. The contact can be controlled by a prescribed load (which is used here) or by a prescribed displacement. Analytical solutions giving the contributions of normal and tangential loading assumed uniform over a single rectangular element will be used. By summation the elastic deflection at each point within and near the contact area will be derived. The elastic displacements are expressed by a double discrete convolution product between influence coefficients and the pressure or 
shear at the contact surface. The normal problem and the tangential problem in partial or gross slip are therefore solved.

The resolution is done by minimizing the complementary energy. An algorithm is developed with the conjugated gradient method. To accelerate the calculation, the Fast Fourier Transforms (FFT) are used to perform the double convolution product. Once the contact problem solved, the strains in the layered half space are calculated.

The solver has since been developed and improved in several ways. Recently, Leroux and Nélias [8] worked on the stick-slip problem for a sphere in contact with a flat half space containing unidirectional cylindrical fibers.

\section{ANISOTROPIC INFLUENCE COEFFICIENTS}

Anisotropic materials are defined by the elastic stiffness tensor $C_{i j k l}$, which satisfies the full symmetry $C_{i j k l}=C_{j i k l}=C_{k l i j}$. The elastic stiffness tensor links stresses to strains with this following relation $\sigma_{\mathrm{ij}}=\mathrm{C}_{\mathrm{ijkl}} \varepsilon_{\mathrm{kl}}$.

The influence coefficients, which link the load to the displacements or to the strains, for an anisotropic material with an anisotropic coating, are obtained with the Green's functions $[1,2]$. These functions can be described explicitly in the Fourier domain, whereas it is more complicated to obtain their formulation in the physical domain due to the general anisotropy of the material [9]. The Fourier domain is therefore used.

A concentrated force (normal or tangential) is applied on the surface of an anisotropic elastic half space. In the absence of body forces, the equations of equilibrium in terms of displacements $u_{k}$ are written as

$$
\mathrm{C}_{\mathrm{ijkl} l} \mathrm{u}_{\mathrm{k}, \mathrm{lj}}=0
$$

Three matrices $3 \times 3$ are defined with the tensor $\mathrm{C}_{\mathrm{ijkl}}$ and the vectors $n$ and $m$, which form a right handed triad with the position vector $\mathrm{x} . \mathrm{Q}, \mathrm{R}$ and $\mathrm{T}$ are a double projection of the elastic stiffness tensor.

$$
Q_{i k}=C_{i j k l} n_{j} n_{l}, R_{i k}=C_{i j k l} n_{j} m_{l}, T_{i k}=C_{i j k l} m_{j} m_{l}
$$

Six distinct eigenvalues $\mathrm{p}$ are obtained by calculating the roots of

$$
\operatorname{det}\left(\mathrm{Q}+\mathrm{p}\left(\mathrm{R}+\mathrm{R}^{\mathrm{T}}\right)+\mathrm{p}^{2} \mathrm{~T}\right)=0
$$

The roots are three pairs of complex conjugates. The complex eigenvectors a of eq. (4) are not a trivial solution. The eigenvectors $b$ are derived by eq. (5)

$$
\begin{gathered}
{\left[Q+p\left(R+R^{T}\right)+p^{2} T\right] \cdot a=0} \\
b=\left(R^{T}+p T\right) \cdot a
\end{gathered}
$$

The vectors $\mathrm{a}$ and $\mathrm{b}$ are the Stroh eigenvectors and $\mathrm{p}$ the associated Stroh eigenvalues [10]. The Green's functions are then expressed as series forms, by superposing the eigensolutions. Three or four terms are enough to solve this complicated problem (the remaining terms are ignored). The first order is obtained with the boundary conditions, then the successive orders are calculated by a recursive form.

\section{APPLICATION TO A LAYERED ANISOTROPIC SPHERE ON FLAT CONTACT}

The influence of the properties of an elastic anisotropic half space with an anisotropic coating which is in contact with a rigid indenter, with a spherical tip, is studied here. The contact parameters, i.e. the contact area and the pressure distribution, will be more specifically investigated. The depth corresponds to direction 3 , which means that the surface is defined by directions 1 and 2 . The subscript $\mathrm{C}$ represents the coating and $\mathrm{S}$ the substrate.

First, materials for the coating are orthotropic and the substrate is cubic (almost isotropic), with the same Poisson's ratio and the same Coulomb's modulus. For the cubic case the Coulomb's modulus is slightly modified relative to an isotropic material, in order to avoid singularities.

The influence of $\mathrm{E}_{\mathrm{C} 1}$ and $\mathrm{E}_{\mathrm{C} 3}$ on the contact pressure distribution is shown in Figs. 1 and 2. The coating thickness is equal to the half of the Hertz radius, $\mathrm{Z}_{\mathrm{C}}=0.5 \mathrm{a}_{\mathrm{Hertz}}$. It is observed that a change of the Young's modulus in the coating along a direction parallel to the surface $\left(\mathrm{E}_{\mathrm{C} 1}\right.$ here) has a moderate effect. The maximum contact pressure is increased by $2 \%$ when $\mathrm{E}_{\mathrm{C} 1}$ is doubled. Moreover it can be observed that the contact area is no more circular. The effect of the Young's modulus in the coating along the depth $\left(\mathrm{E}_{\mathrm{C} 3}\right)$ is more pronounced: increase of $14 \%$ of the maximum contact pressure and decrease of the contact radius by $8 \%$ when the Young's modulus is increased by a factor 2 .

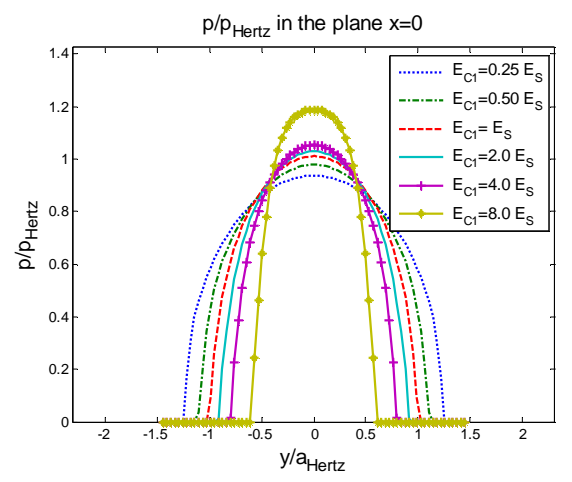

Figure 1: Influence of $E_{C 1}$ on the contact pressure

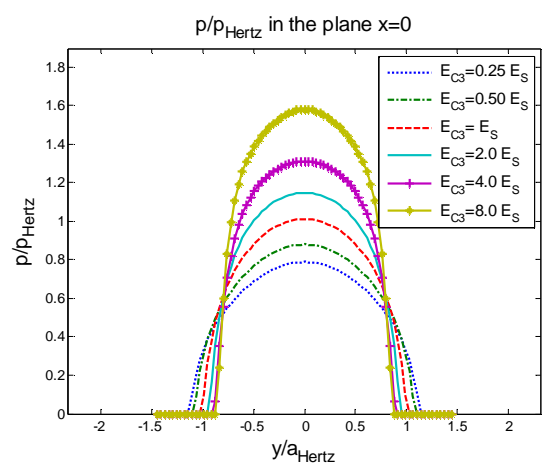

Figure 2: Influence of $\mathrm{E}_{\mathrm{C3}}$ on the contact pressure 
The effect of the coating thickness is shown in Fig. 3. Here, the Young's Modulus of the coating in direction 3 is equal to $\mathrm{E}_{\mathrm{C} 3}=2 \mathrm{E}_{\mathrm{S}}$. The case $\mathrm{Z}_{\mathrm{C}}=0 \mathrm{a}_{\mathrm{Hert}}$ corresponds to an half space without coating and the case $Z_{C}=\infty a_{\text {Hertz }}$ to the half space composed only with the coating. When the depth of the coating increases, the influence of the coating become predominant relative to the influence of the substrate. As $E_{C 3}>E_{S}$, the maximum contact pressure increases with the depth.

Now roles are reversed, the coating is cubic, almost isotropic, and materials which defined the substrate are orthotropic.

The influence of $E_{\mathrm{S} 1}$ and $\mathrm{E}_{\mathrm{S} 3}$, the Young's modulus of the substrate, on the contact pressure distribution is studied in Figs. 4 and 5 . The coating thickness is equal to the half of the Hertz radius, $\mathrm{Z}_{\mathrm{C}}=0.5 \mathrm{a}_{\mathrm{Hertz}}$. The effect of $\mathrm{E}_{\mathrm{S} 1}$ is limited. When $\mathrm{E}_{\mathrm{S} 1}$ is doubled, the maximum pressure increases by $0.7 \%$. Conversely the Young's modulus of the substrate along the depth, $\mathrm{E}_{\mathrm{S} 3}$, has a strong influence. The maximum pressure is $13 \%$ higher when $\mathrm{E}_{\mathrm{S} 3}$ is multiplied by 2 .

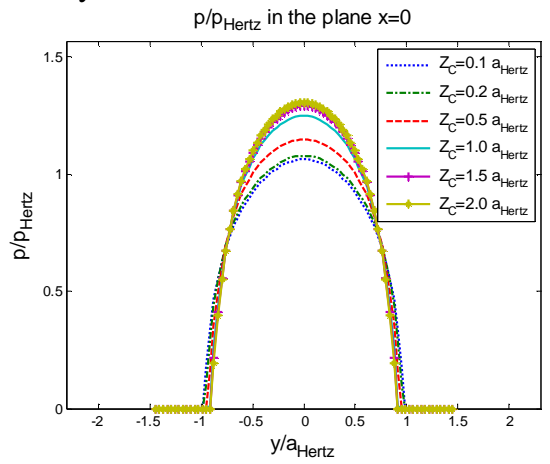

Figure 3: Influence of $\mathrm{Z}_{\mathrm{C}}$ on the contact pressure, $\mathrm{E}_{\mathrm{C} 3}=\mathbf{2} \mathrm{E}_{\mathrm{S}}$

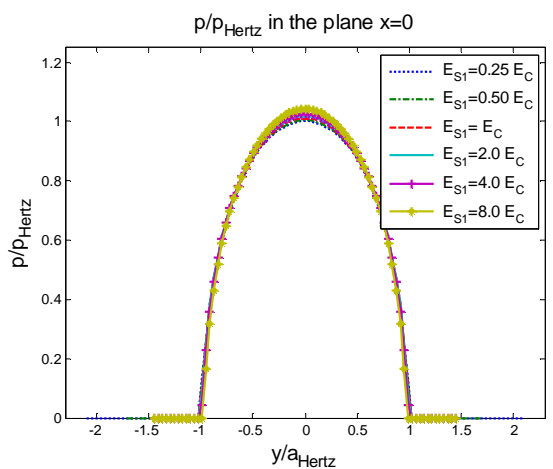

Figure 4: Influence of $E_{S 1}$ on the contact pressure

Figure 6 shown the effect of the isotropic coating thickness on an anisotropic substrate, for the pressure distribution. The Young's modulus of the substrate in direction 3 is equal to $\mathrm{E}_{\mathrm{S} 3}=2 \mathrm{E}_{\mathrm{C}}$. As $\mathrm{E}_{\mathrm{C}}<\mathrm{E}_{\mathrm{S} 3}$, the maximum contact pressure decreases with the depth. It can be observed that from $Z_{C}>2 a_{\text {Hertz }}$, the difference between the maximum pressure of the isotropic half space and the layered half space is less than $1 \%$, the coating erases the anisotropy effect of the substrate.

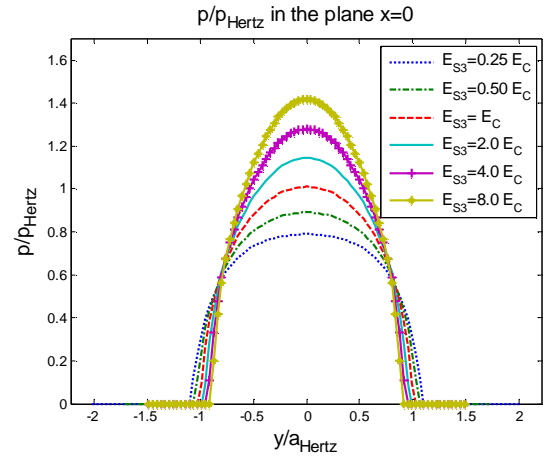

Figure 5: Influence of $\mathrm{E}_{\mathrm{S3}}$ on the contact pressure

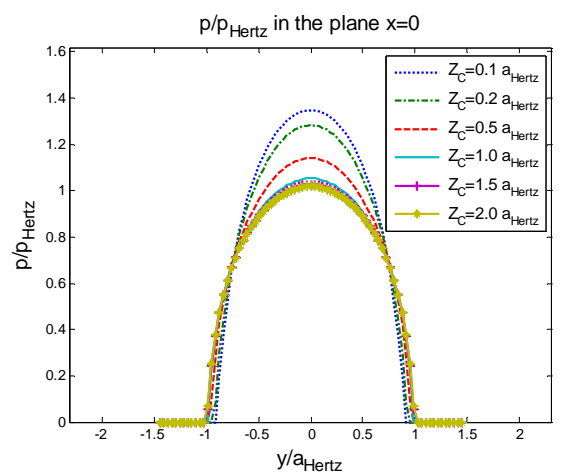

Figure 6: Influence of $Z_{C}$ on the contact pressure, $E_{S 3}=2 E_{C}$

\section{CONCLUSION}

A semi analytical method has been developed for the contact problem of anisotropic elastic materials with an anisotropic coating, by using the Green's functions. The model has been validated by comparison with the results of O'Sullivan for isotropic materials with an isotropic coating and by comparison with a FE model for anisotropic half spaces.

Parametric studies have been done for anisotropic coating on an isotropic substrate and for the reversed case, anisotropic substrates with an isotropic coating. It is found that the stiffness along the normal to the contact, in the coating or in the substrate, has a strong influence on the contact solution in terms of pressure distribution and contact size. Conversely a change of the Young's modulus along a direction parallel to the surface (plane $(1,2)$ ) does not significantly affect the contact pressure distribution but the contact area is no more circular, it becomes elliptic. Finally it should be emphasized that, when the coating thickness exceeds the contact radius, the effect of the substrate anisotropy vanishes quickly.

\section{ACKNOWLEDGMENTS}

This work is supported by the French FUI Project Innolub.

\section{REFERENCES}

[1] Yang, B., Pan, E., 2002, "Three-dimensional Green's functions in anisotropic trimaterials," International Journal of Solids and Structures, 39, pp. 2235-2255. 
[2] Ting, T.C.T., and Ven-Gen Lee, 1997, "The treedimensional elastostatic Green's function for general anisotropic linear elastic solids," Mech. appl. Math., 50, pp. 407-426.

[3] Bagault, C., Nélias, D., Baietto, M.-C., 2012, "Contact analyses for anisotropic half space: effect of the anisotropy on the pressure distribution and contact area," Journal of Tribology, in press.

[4] Pan, E., and Yuan, F.G., 2000, "Three-dimensional Green's functions in anisotropic bimaterials," International Journal of Solids and Structures, 37, pp. 5329-5351.

[5] He, L., and Ovaert, T.C., 2008, "Three-dimensional rough surface contact model for anisotropic materials," Journal of Tribology, 130.

[6] Johnson, K.L., 1985, "Contact mechanics," Cambridge university press, London.
[7] Jacq, C., Nélias, D., Lormand, G., and Girodin, D., 2002, "Development of a three-dimensional semianalytical elastic-plastic contact code," Journal of Tribology, 124, pp. 653-667.

[8] Leroux, J., and Nélias, D., 2011, "Stick-slip analysis of a circular point contact between a rigid sphere and a flat unidirectional composite with cylindrical fibers," International Journal of Solids and Structures, 48, pp. 3510-3520.

[9] Ting, T.C.T., 1996, "Anisotropic elasticity: theory and applications," Oxford university press, Oxford.

[10] Ting, T.C.T., 1997, "New explicit expression of BarnettLothe tensors for anisotropic linear elastic materials," Journal of Elasticity, 47, pp. 23-50. 